Reprod. Nutr. Dévelop., 1985, 25 (1 A), 101-111.

\title{
Influence de la durée d'éclairement sur la production et la fécondance des spermatozoïdes chez le bélier adulte lle-de-France
}

\author{
G. COLAS, Y. GUÉRIN, V. CLANET, Aline SOLARI (*)
}

Station de Physiologie de la Reproduction, I.N.R.A., Nouzilly, 37380 Monnaie, France.

(*) Station centrale de Physiologie animale, I.N.R.A., 78350 Jouy-en-Josas.

Summary. Effect of daylength on the production and fertilizing ability of spermatozoa in the lle-de-France ram.

Two groups, A and B, of 5 adult lle-de-France rams each of the same age were exposed for 52 weeks (October 1975 to October 1976) to an artificial light regime reproducing natural variations of daylength (DL). In group $A$, DL increased from 8 to $16 \mathrm{~h}$ during the first 26 weeks and then decreased. In group B, DL varied in an opposite manner. Light intensity was constant $\left(300 \mathrm{lux} / \mathrm{m}^{2}\right)$ and ambient temperature was controlled $\left(20 \pm 4^{\circ} \mathrm{C}\right)$.

The rams were collected using an artificial vagina twice weekly $(2 \times 2$ ejaculates/ male/week) during the whole experimental period (EP). The volume and concentration of each ejaculate were recorded. The $\mathrm{pH}$ of the raw semen was also measured on the first ejaculate once a week or every two weeks, according to material possibilities.

The unselected ejaculates of all rams were used for artificial insemination (Al) on the same days in the same flocks between January and October 1976. Al (980 ewes) was always carried out after oestrus synchronization (FGA + PMSG). Amongst these ejaculates, some ( 3 ejaculates/male/group/period) could be used for both in vitro $(\mathrm{pH})$ and in vivo (Al) measurements :

- Weekly sperm production (SP) varied in an opposite manner in both groups during the whole EP. In group $A$, minimal $\left(10.6 \pm 4.0 .10^{9}\right)$ and maximal $\left(25.5 \pm 5.1 .10^{9}\right)$ levels were reached from weeks 12 to 28 and weeks 38 to 52, respectively. In group B, SP was maximal $\left(21.8 \pm 2.3 .10^{9}\right)$ from weeks 9 to 24 and minimal $\left(12.7 \pm 5.2 .10^{9}\right)$ from weeks 36 to 49 .

The differences between rams were significant within periods of high and low production. versa.

- The sperm $\mathrm{pH}$ was somewhat higher in both groups when $\mathrm{DL}$ increased and vice-

Fertility after Al was higher with rams exposed to short days than with rams exposed to long days (65.7 vs $56.7 \% ; \mathrm{P}<0.01)$. The season of Al had no effect on mean fertility (61.0 vs $62.3 \%$ for ewes inseminated from January to June and from July to October, respectively), whatever the $D L$.

No correlation was found between the $\mathrm{pH}$ of the sperm and its fertilizing ability.

From January to April, a highly significant correlation existed between fertility and prolificacy in the 10 rams $(r=+0.607)$.

These results show that photoperiodism has an important effect on both ejaculated sperm production and fertility in rams. Ram reproductive performance can thus be much improved in spring using an artificial light regime 6 months of decreasing light, 6 months of increasing light) whose daylength must begin to decrease 9 to 12 weeks earlier. 


\section{Introduction.}

De nombreux travaux ont mis en évidence l'importance du photopériodisme dans le contrôle de l'activité testiculaire chez le bélier (Ortavant, 1958, 1977 ; Pelletier, 1971 ; Lincoln et Davidson, 1977 ; etc...). Ils permettent de penser qu'en modifiant l'environnement lumineux, il doit être possible, dans cette espèce, d'améliorer les capacités de reproduction du mâle à certaines époques de l'année, en particulier au printemps. Quelques expériences ont déjà été effectuées dans ce domaine (Alberio, 1976 ; Alberio et Colas, 1976; Schanbacher, 1979; Tulley et Burfening, 1983). Les réponses apportées ne permettent pas toutefois de connaître avec précision l'évolution de la production spermatique et la fécondance réelle des gamètes chez des animaux adultes soumis à un environnement lumineux contrôlé pendant de longs mois.

Or, à l'heure actuelle, on est de plus en plus souvent amené à solliciter les béliers au printemps lorsque leur aptitude sexuelle est fortement diminuée. C'est le cas de certains centres d'insémination artificielle qui, en raison de l'utilisation croissante des progestagènes pour le contrôle des cycles sexuels, réalisent l'essentiel de leur activité à cette époque de l'année.

La présente étude a pour but d'apporter quelques nouveaux éléments dans ce domaine.

\section{Matériel et méthodes.}

\section{A. Animaux expérimentaux.}

a) Mâles. - 2 groupes (A et $B$ ) de 5 béliers adultes ( 21 mois en début d'expérience) lle-de-France, nés en février, ont été utilisés pendant un an (octobre 1975 à octobre 1976). A l'intérieur de chaque groupe, les animaux n'avaient entre eux aucun lien de parenté.

b) Femelles. -980 femelles de plusieurs races et d'âge variable ( $\geq 1$ an) ont servi à mesurer le pouvoir fécondant du sperme des 10 béliers. Elles appartenaient, dans leur très grande majorité, à des élevages privés situés dans un rayon d'environ $150 \mathrm{~km}$ du laboratoire.

\section{B. Traitement photopériodique.}

Après une période d'adaptation de 6 mois à leur nouvel environnement, les béliers ont été soumis pendant 52 semaines à un régime lumineux reproduisant les variations naturelles de la durée d'éclairement sous notre latitude.

- Groupe A : Augmentation de la durée d'éclairement (DE) de 8 h (16 h obscurité) à $16 \mathrm{~h}$ ( $8 \mathrm{~h}$ obscurité) pendant les 26 premières semaines, diminution (16 h-8 h) ensuite.

- Groupe B : évolution inverse.

La DE était modifiée d'un quart d'heure tous les six jours.

Les béliers vivaient dans deux cellules $(3,1 \mathrm{~m} \times 2,80 \mathrm{~m} \times 4,0)$ identiques, étanches à la lumière, ventilées et protégées des températures extérieures $\left(20 \pm 4^{\circ} \mathrm{C}\right)$. L'intensité lumineuse était de $300 \mathrm{lux} / \mathrm{m}^{2}$ à environ 1 mètre du sol.

La ration alimentaire était la même pour les deux groupes de béliers. 
C. Contrôles.

a) Production quantitative de sperme. - Les béliers ont été collectés à l'aide d'un vagin artificiel deux fois par semaine $(2 \times 2$ éj./mâle/sem. $)$ pendant 52 semaines.

Le nombre de spermatozoïdes recueillis était déterminé à partir du volume là $0,05 \mathrm{ml}$ près) et de la concentration (mesurée par néphélométrie, taux de dilution $1 / 400$ ) de l'éjaculat. La production hebdomadaire de chaque animal était donc égale à la somme des 4 produits obtenus.

b) Qualité des gamètes.

- In vitro. - Les examens de laboratoire étaient réalisés sur le premier éjaculat, le même jour pour les lots $A$ et $B$ :

- pourcentage d'anomalies morphologiques : déterminé toutes les deux semaines selon une méthode déjà décrite (Colas, 1980). Ce contrôle n'a été effectué que pendant la première période (26 premières semaines). Les résultats ont déjà fait l'objet d'une publication (Colas, 1983) ;

- $\mathrm{pH}$ du sperme pur, mesuré une fois par semaine ou une fois tous les quinze jours selon les possibilités matérielles, dans les deux à trois minutes qui suivaient le recueil de l'éjaculat.

La motilité massale et le pourcentage de spermatozoïdes colorés à l'éosinenigrosine n'ont pas été contrôlés puisqu'ils ne sont pas reliés à la fécondance (Colas, 1981).

- In vivo. - Les contrôles de fécondance se sont déroulés entre janvier et octobre 1976 : les éjaculats non triés de tous les béliers étaient mis en place le même jour dans le même troupeau par le même opérateur.

Les brebis étaient traitées à l'acétate de fluorogestone (30 mg par éponge au cours de l'anoestrus saisonnier, $40 \mathrm{mg}$ pendant la saison sexuelle) et à la PMSG. Les doses de PMSG variaient (400 à $650 \mathrm{UI}$ ) selon la race, la saison et l'état physiologique de la brebis (Cognié et al., 1975).

Les femelles étaient inséminées avec du sperme préparé et conservé $\left(+15^{\circ} \mathrm{C}\right)$ selon une méthode déjà publiée (Colas, Brice et Guérin, 1974).

Parmi les éjaculats utilisés in vivo, certains ( 3 éj. / mâle et par période) avaient pu faire l'objet d'une mesure de $\mathrm{pH}$. A l'intérieur de chaque période, les trois mesures ont été effectuées les mêmes jours sur les 10 béliers.

\section{Expression et analyse des résultats.}

Les productions spermatiques hebdomadaires moyennes de chaque groupe d'animaux ont été représentées par leur moyenne affectée de l'écart-type de la moyenne $(\sigma / \sqrt{5})$. Les productions minima ou maxima par animal sont comparées par analyse de variance.

La fécondance du sperme est exprimée en pourcentage de mise bas, la prolificité par le nombre d'agneaux nés, vivants ou morts, pour 100 femelles mettant bas. La comparaison de la fédondance des différents béliers à l'intérieur d'un même groupe et pour une même DE a été effectuée par l'analyse du $\chi^{2}$. De même, les effets de la DE et de la saison d'I.A. ont été analysés par le test du $\chi^{2}$ corrigé de Yates. 
Les corrélations entre $\mathrm{pH}$ et taux d'agnelage ont été calculées pour chaque groupe de béliers, non pas à partir des données individuelles relatives à chaque éjaculat, mais à partir de la moyenne des mesures $(n=3)$ effectuées sur chaque bélier, le nombre de femelles inséminées par éjaculat étant trop faible.

\section{Résultats.}

A. Production spermatique.

L'évolution de la production spermatique est représentée sur la figure 1. Les moyennes minimales et maximales figurent dans le tableau 1.

Comme on peut le constater, les deux courbes représentées sur la figure 1 ont un profil opposé tout au long de la période expérimentale.

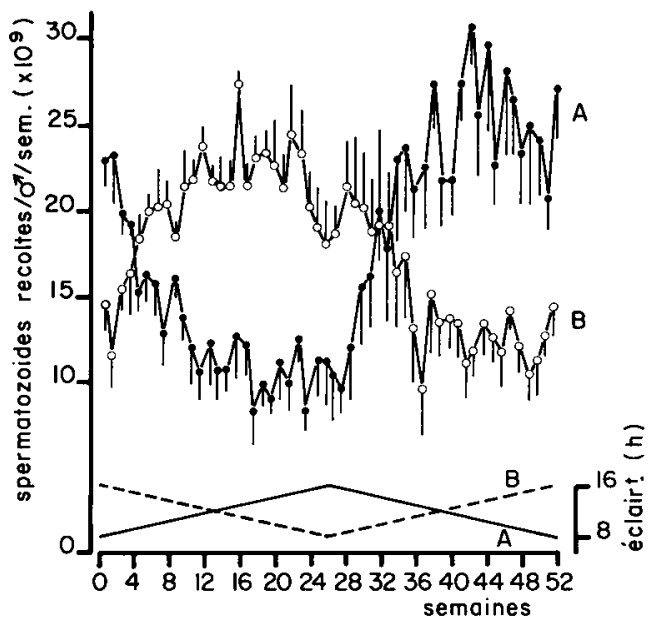

FIG. 1. - Influence de la durée d'éclairement artificiel sur la production spermatique hebdomadaire $(\mathrm{m} \pm \sigma / \sqrt{\mathrm{n}}) d u$ bélier adulte /le-de-France $\left(\mathrm{n}=5,2 \times 2\right.$ éj./ $\sigma^{*} / \mathrm{sem} .$, to $=$ octobre 1975).

TABLEAU 1

Production spermatique hebdomadaire ( $\times 10^{9} \mathrm{spz}$ ) minimum et maximum selon la durée d'éclairement chez le bélier lle-de-France $(\mathrm{n}=5)$.

\begin{tabular}{ccc}
\hline Animaux & Production minimum & Production maximum \\
\hline \multirow{2}{*}{ Groupe A } & $\mathrm{S}_{12}$ à $\mathrm{S}_{28}$ & $\mathrm{~S}_{38}$ à $\mathrm{S}_{52}$ \\
& $10,6 \mathrm{NS}+0$ & $25,5 \pm 5,1$ \\
Groupe B & $\mathrm{NS}_{36}$ à $\mathrm{S}_{49}$ & $\mathrm{NS}_{9}$ à $\mathrm{S}_{24}$ \\
& $12,7 \pm 5,2$ & $21,8 \pm 2,3$ \\
\hline
\end{tabular}

S : Semaine ; NS : Non significatif. 
Chez les béliers soumis à une durée d'éclairement croissante (Groupe A), la quantité de spermatozoïdes recueillis diminue rapidement pendant les 12 premières semaines. Elle se stabilise ensuite à un niveau minimum $\left(10,6 \pm 4,0 \times 10^{9} \mathrm{spz}\right.$. $)$ jusqu'à la $28^{\mathrm{e}}$ semaine et augmente à nouveau (141,5\%) pour conserver une valeur élevée $\left(25,6 \pm 5,1 \times 10^{9} \mathrm{spz}\right.$. $)$ de la $38^{\mathrm{e}}$ à la $52^{\mathrm{e}}$ semaine.

Chez les béliers du lot $B$, les moyennes hebdomadaires sont maxima $\left(21,8 \pm 2,3 \times 10^{9} \mathrm{spz}\right.$.) de la 9 e à la 24 e semaine et minima $\left(12,7 \pm 5,2 \times 10^{9} \mathrm{spz}\right.$.) de la $36^{e}$ à la $49^{e}$ semaine. Dans ce cas, la variation n'est que de $70,6 \%$.

A l'intérieur de ces périodes, les différences de production entre animaux sont significatives.

B. $p H$ du sperme.

Le $\mathrm{pH}$ varie selon la durée d'éclairement ; il est plus élevé dans les deux lots lorsque les jours augmentent et inversement. Toutefois, son évolution est différente selon les lots puisque les valeurs moyennes sont supérieures à 7 pendant 28 semaines $\left(S_{6}\right.$ à $\left.S_{33}\right)$ dans le lot $A$ et pendant 11 semaines seulement $\left(S_{38}\right.$ à $\left.S_{48}\right)$ dans le lot B (fig. 2).

C. Fécondance.

a) Influence de la durée d'éclairement. - Dans l'ensemble (tabl. 2), la fécondance du sperme est meilleure en jours courts qu'en jours longs $(65,7$ vs $56,7 \%$ ) $(P<0,01)$.

Pendant la première partie de l'expérience, les taux de fertilité sont plus élevés chez les béliers du lot $B$ (lumière décroissante) que chez ceux du lot $A$

\section{TABLEAU 2}

Effet de la durée artificielle d'éclairement sur la fécondance

(\% M.B.) du sperme de bélier (éjaculats non triés, 5 mâles/groupe).

\begin{tabular}{|c|c|c|c|c|}
\hline \multirow{2}{*}{$\begin{array}{l}\text { Durée } \\
\text { éclairement } \\
\text { des mâles }\end{array}$} & \multicolumn{3}{|c|}{ Groupe } & \multirow{2}{*}{ Total } \\
\hline & & A & B & \\
\hline Croissante & $\begin{array}{c}\text { ९ (a) } \\
\% \text { M.B. }\end{array}$ & $\begin{array}{c}214 \\
1 \\
51,9 \\
(40,6 \text { à } 64,1)\end{array}$ & $\begin{array}{c}253 \\
3 \\
60,9 \\
(56,5 \text { à } 64,3)\end{array}$ & $\begin{array}{c}467 \\
5 \\
56,7\end{array}$ \\
\hline Décroissante & $\begin{array}{c}\text { Q (a) } \\
\% \text { M.B. }\end{array}$ & $\begin{array}{c}277 \\
2 \\
65,3 \\
(58,9 \text { à } 74,2)\end{array}$ & $\begin{array}{c}236 \\
4 \\
66,1 \\
(58,3 \text { à } 70,4)\end{array}$ & $\begin{array}{c}513 \\
6 \\
65,7\end{array}$ \\
\hline
\end{tabular}

(a) : Femelles inséminées (500 × $10^{6} \mathrm{spz}$.) après induction de l'œestrus par FGA + PMSG.

(1): Pourcentage de fertilité minimum et maximum.

$\left.\begin{array}{l}1 \text { et } 4 \\ 2 \text { et } 3\end{array}\right\}$ Sperme mis en place les mêmes jours dans les mêmes élevages.

1 vs $4: P<0,01\left(X^{2}=19,61\right)$.

1 vs $2: \mathrm{P}<0,01\left(\mathrm{X}^{2}=8,54\right)$.

5 vs $6: \mathrm{P}<0,01\left(\mathrm{X}^{2}=7,88\right)$.

2 vs 3, 3 vs 4, 1 vs 3 : NS. 
(lumière croissante) $(66,1$ vs 51,$9 ; P<0,01)$. Au-delà, la tendance est la même (4,3 points d'écart) mais la différence n'est pas significative.

II existe aussi (tabl. 2) des différences de résultat entre individus, plus importantes en jours longs qu'en jours courts mais dans aucune de ces deux périodes, elles ne sont significatives.

b) Influence de la saison. - Les résultats enregistrés dans les 18 élevages ont été analysés en fonction de l'époque d'insémination ( Printemps " = janvier à juin, "Automne " = juillet à octobre). On voit (tabl. 3) que la saison n'a pas d'effet sur la fertilité moyenne $(61,0 \%$ au $P, 62,3 \%$ en $A ; P>0,05)$ et ce, quelle que soit la durée d'éclairement. Les pourcentages de mises-bas sont par contre plus élevés après insémination à l'aide de sperme récolté pendant les jours décroissants qu'après insémination avec du sperme récolté pendant les jours croissants mais la différence n'est significative qu'au printemps $\langle 56,7$ vs $65,2 \%$; $P<0,01)$.

c) Influence du $p H$. - Aucune corrélation significative n'a été trouvée entre le $\mathrm{pH}$ moyen du sperme pur de chacun des 10 béliers et sa fécondance ( $r=+0,543$ et $r=+0,09$ pour chacune des deux périodes).

\section{Prolificité.}

A aucun moment, les différences de prolificité ne sont significatives, qu'il s'agisse de l'effet de la durée d'éclairement ou de la saison d'insémination.

Entre la $13^{\mathrm{e}}$ et la $26^{\mathrm{e}}$ semaine, il existe une corrélation hautement significative entre les taux de fertilité et de prolificité des 10 béliers $(r=+0,607)$.

TABLEAU 3

Effet de la saison d'insémination sur la fécondance du sperme de bélier selon la durée d'éclairement (éjaculats non triés).

\begin{tabular}{cccc}
\hline \multirow{2}{*}{$\begin{array}{c}\text { Durée } \\
\text { éclairement } \\
\text { des mâles }\end{array}$} & \multicolumn{2}{c}{ Saison d'insémination } & \multirow{2}{*}{ Total } \\
\cline { 2 - 3 } & Printemps & Automne & \\
\hline Croissante & 1 & 2 & 5 \\
& 56,7 & 56,9 & 56,7 \\
& $(323)$ & $(144)$ & $(467)$ \\
Décroissante & 3 & 4 & 6 \\
& 65,2 & 66,7 & 65,7 \\
Total & $(336)$ & $(177)$ & $(513)$ \\
\hline & 61,0 & 62,3 & \\
\hline
\end{tabular}

( ) : Brebis inséminées $\left(500 \times 10^{6} \mathrm{spz} . /\right.$ O $)$ après induction des chaleurs par FGA + PMSG.

1 vs $3: \mathrm{P}<0,01\left(\mathrm{X}^{2}=6,66\right)$.

1 vs $4: 0,01<P<0,05\left(x^{2}=4,66\right)$.

5 vs $6: \mathrm{P}<0,01\left(\mathrm{X}^{2}=7,88\right)$.

( 2 vs 3,2 vs $4=$ NS). 


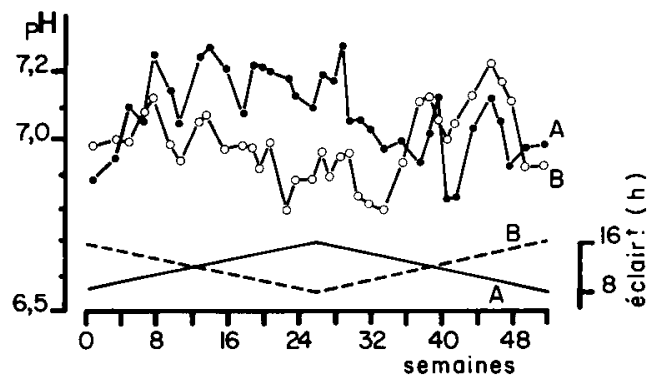

FIG. 2. - Influence de la durée d'éclairement artificiel sur le pH du sperme pur chez le bélier adulte lle-de-France $(\mathrm{n}=5)$.

\section{Discussion.}

\section{A. Production spermatique.}

Comme on pouvait s'y attendre (Ortavant et Thibault, 1956 ; Alberio, 1976), la durée d'éclairement modifie très fortement la production spermatique chez le bélier adulte.

Le fait que l'on obtienne au même moment des valeurs opposées chez des animaux d'âge identique soumis à des traitements lumineux inversés, prouve que les variations enregistrées ne sont dues qu'au photopériodisme. On peut donc stimuler de façon efficace et durable la fonction testiculaire dans cette espèce à une période de l'année où normalement la production spermatique quantitative et qualitative sont faibles. Un tel résultat présente un intérêt pratique évident notamment pour les Centres d'Insémination qui vont ainsi pouvoir faire coïncider la période de forte production de semence avec celle où les mâles sont le plus sollicités. II existe d'ailleurs d'autres schémas que celui que nous avons étudié pour augmenter l'activité sexuelle chez le bélier. Ortavant et Thibault (1956), Sanford et al. (1978) reproduisent en six mois le cycle annuel d'éclairement. Lincoln (1976, 1977), Langford et Marcus (1982) utilisent alternativement des périodes à jours longs $(16 \mathrm{~h} / 8 \mathrm{~h})$ et des périodes à jours courts $(8 \mathrm{~h} / 16 \mathrm{~h})$, chacune d'elles ayant une durée de 12 à 17 semaines. On peut enfin, comme l'a réalisé Ortavant (1977), éclairer les animaux à deux reprises au cours du nychtémère : pendant $7 \mathrm{~h}$ consécutives puis de 16 à $17 \mathrm{~h}$ après l'aube artificielle. Toutefois, avant de mettre en pratique de tels modèles expérimentaux, il est indispensable de vérifier si leur effet est bien répétable d'une année sur l'autre.

Lorsque la durée d'éclairement décroît, le nombre de spermatozoïdes recueillis augmente de 70 à $114 \%$ selon les lots $B$ ou $A$. Ces chiffres sont inférieurs à ceux que Dacheux et al. (1981) obtiennent $(+370 \%)$ en canulant le canal efférent chez des sujets de même race exposés à la lumière naturelle entre juin et décembre. En fait, ce sont surtout les productions quotidiennes maxima qui sont plus faibles dans notre expérience $\left(3,6\right.$ ou $3,1 \times 10^{9} \mathrm{spz}$./jour pour les lots $B$ ou A contre 4,8 $\times 10^{9} \mathrm{spz}$./jour). En photopériode croissante, en effet, les moyennes sont de 1,5 et $1,8 \times 10^{9} \mathrm{spz}$. contre $1,02 \times 10^{9} \mathrm{spz}$. pour les animaux canu- 
lés. Pendant la période de moindre activité sexuelle (jours croissants), le rythme de collecte est donc proche du rythme maximum possible. Lorsque les jours diminuent par contre, on peut augmenter la fréquence des prélèvements. Nous avons montré sur la race Romanov (Colas et al., 1975), que le fait de passer de 4 à 6 éjaculats par semaine - ce que les animaux supportent très bien - permet de multiplier par 1,5 le nombre moyen de spermatozoïdes recueillis. Si l'on applique ce coefficient à la production maximale de nos béliers, on voit que le niveau atteint est proche $\left(4,6 \times 10^{9} \mathrm{spz}\right.$.), voire supérieur $\left(5,4 \times 10^{9} \mathrm{spz}\right.$.), à celui rapporté par les auteurs précités.

L'analyse statistique révèle de grandes différences individuelles pendant les périodes de basse et de haute production. Les animaux répondent donc de façon inégale aux stimulations lumineuses. Certains y sont très sensibles, d'autres, paraissent peu affectés par la durée d'éclairement. Pour ces derniers, il est donc inutile d'envisager un traitement photopériodique. Dans la pratique, leur détection pourrait être fondée sur le contrôle de l'évolution de la taille testiculaire, fortement liée à la production gamétique (Ortavant, 1958 ; Colyer, 1970 ; Amann, 1970), puisque chez de tels sujets, les variations pondérales du testicule sont faibles.

Le nombre de spermatozoïdes récoltés atteint son maximum de 9 à 12 semaines, selon les lots, après l'inversion du rythme lumineux. De tels délais sont supérieurs aux durées d'élaboration de la cellule dans la gonade (Ortavant, 1958) et de son transit épididymaire (Amir et Ortavant, 1968). Cette différence est cependant normale si l'on songe, d'une part, à l'imprécision avec laquelle on peut dater une production par éjaculation spontanée, d'autre part, au temps de latence qui s'écoule toujours entre la mise en route d'un processus testiculaire et sa pleine expression au niveau de la semence.

\section{B. Qualité des gamètes.}

a) Anomalies morphologiques. - Nous avons déjà montré à propos de cette expérience (Colas, 1983), que le pourcentage de spermatozoïdes anormaux était, au cours des 26 premières semaines, plus élevé en jours longs qu'en jours courts. Les taux d'élimination des éjaculats ( $>20 \%$ d'anomalies morphologiques) sont respectivement de $41,5 \%$ et 12,3 ( $n=65$ ). Or, en milieu naturel, lorsque les jours augmentent, la morphologie du gamète mâle subit aussi des altérations profondes (Colas, 1980 ; Mickelsen, Paisley et Dahmen, 1981). On voit donc l'intérêt que présente, sur le plan de la qualité de la semence, le conditionnement lumineux dans cette espèce.

b) $p H$. - Le $\mathrm{pH}$ du sperme pur a tendance à s'alcaliniser lorsque la DE augmente et à s'acidifier ensuite. Il suit donc une évolution inverse de celle qui a été observée pour la concentration de l'éjaculat par Alberio (1976), Barrell et Lapwood (1979), Neves, Günzel et Schmidt (1980). Ce phénomène reflète vraisemblablement les variations de production d'acide lactique, elle-même fonction du nombre de spermatozoïdes par unité de volume et aussi celles de l'activité glycolytique cellulaire comme l'a montré Mann (1964).

Nous n'avons pas réussi à mettre en évidence de corrélation significative entre les valeurs obtenues et la fertilité. Cette absence de résultat ne doit pas sur- 
prendre puisque dans la littérature, aucun auteur n'a recours à cette méthode pour contrôler, de manière précise, la qualité de la semence.

c) Fécondance. - La durée d'éclairement modifie beaucoup la fécondance du sperme. Ce résultat va dans le sens des observations faites par plusieurs auteurs en milieu naturel (Salamon et Robinson, 1962 ; Fowler, 1965 ; Colas et Brice, 1976 ; Colas, 1981).

Les différences de fertilité enregistrées au cours de chaque période entre les lots $A$ et $B$ ne sont bien dues qu'à la qualité de la semence puisque les comparaisons sont établies dans les mêmes élevages, avec des nombres de spermatozoïdes par femelles identiques et sans aucun tri d'éjaculats. Elles reflètent la véritable influence du photopériodisme sur la qualité spermatique du bélier lle-de-France.

Les écarts obtenus sont inférieurs aux chiffres publiés par Schanbacher (1979) en saillie naturelle. Ils s'expliquent sans doute par le fait qu'en choisissant ce mode d'accouplement, l'auteur fait intervenir plusieurs facteurs : la libido du mâle, la qualité du sperme et le nombre de spermatozoïdes par femelle.

Les taux de mises-bas enregistrés après insémination artificielle au printemps, période d'anoestrus chez la brebis, et en automne sont comparables (tabl. 3). En utilisant des béliers artificiellement mis en saison sexuelle par un conditionnement lumineux, il est donc possible d'atteindre des niveaux de fertilité voisins toute l'année.

\section{Relation entre prolificité et fertilité.}

L'existence d'une corrélation positive et significative entre le pourcentage de mises-bas et la prolificité prouve que le bélier exerce un effet direct sur le nombre d'agneaux par mise-bas. Des résultats comparables ont déjà été obtenus dans la même espèce par Lees (1971), Schanbacher (1979), Despierre et al. (1981) cité par Colas et al. (1984) après accouplement naturel ou artificiel. Chez les porcins, Ollivier et Legault (1967) ont observé le même phénomène et trouvé une différence de 5,2 porcelets à la naissance entre le verrat le plus prolifique et le verrat le moins prolifique.

Pour interpréter une telle relation, on peut penser que, par la qualité de sa semence, le mâle agit à la fois sur le nombre d'ovocytes fécondés et sur leur devenir dans l'utérus. Fournier-Delpech et al. (1979) ont démontré que la mortalité embryonnaire dépend du stade de maturation des spermatozoídes dans l'épididyme. On sait, par ailleurs, que chez le taureau (Bearden et al., 1956 ; Courot et Tourneur, 1976), cette mortalité est d'autant plus faible que le taux de fécondation est plus élevé. L'influence du reproducteur va donc bien au-delà de ce que l'on estime généralement.

\section{Conclusion.}

La production spermatique éjaculée dépend étroitement du photopériodisme chez le bélier lle-de-France. Au cours d'un cycle lumineux annuel, elle augmente lorsque la durée d'éclairement diminue et inversement. On peut donc modifier la saison sexuelle $d^{\prime} u n$ reproducteur par cette méthode. 
Le $\mathrm{pH}$ du sperme pur est plutôt alcalin en jours longs, acide en jours courts.

Le pouvoir fécondant du sperme dilué et conservé quelques heures à $+15^{\circ} \mathrm{C}$ est significativement plus faible en jours longs (lumière croissante) qu'en jours courts (lumière décroissante).

Reçu en juin 1984.

Accepté en septembre 1984.

Remerciements. - Les auteurs tiennent à remercier Messieurs Courot et Ortavant pour leurs commentaires sur cette étude.

\section{Références}

ALBERIO R., 1976. Rôle de la photopériode dans le développement de la fonction de reproduction chez l'agneau lle-de-France, de la naissance à 21 mois. Th. Doct. $3^{e}$ cycle, Univ. Paris VI, $57 \mathrm{pp}$.

ALBERIO R., COLAS G., 1976. Influence of photoperiodism on the sexual development of the young Ile-de-France ram. VIIIth. int. Congr. Anim. Reprod. \& A.I., vol. III, Krakow, 26-29.

AMANN R. P.,1970. Sperm production rates, 433-482. In A. D. JOHNSON, W. R. GOMES and N. L. VAN DEMARK. The testis. Acad. Press, New York and London.

AMIR D., ORTAVANT R., 1968. Influence de la fréquence des collectes sur la durée de transit des spermatozoïdes dans le canal épididymaire du bélier. Ann. Biol. anim. Bioch. Biophys., 8, 195-207.

BARRELL G. K., LAPWOOD K. R., 1979. Seasonality of semen production and plasma luteinizing hormone, testosterone and prolactin levels in Romney, Merino and Polled dorset rams. Anim. Reprod. Sci., 1, 213-228.

BEARDEN H. J., HANSEL W. M., BRATTON R. W., 1956. Fertilization and embryonic mortality rates of bulls with histories of either low or high fertility in artificial breeding. J. Dairy Sci., 39, 312-318.

COGNIE Y., HERNANDEZ-BARRETO M., SAUMANDE J., 1975. Low fertility in nursing ewes during the non-breeding season. Ann. Biol. anim. Bioch. Biophys., 15, 329-343.

COLAS G., 1980. Variations saisonnières de la qualité du sperme chez le bélier lle-de-France. I. Etude de la morphologie cellulaire et de la motilité massale. Reprod. Nutr. Dévelop., 20, 1789-1799.

COLAS G., 1981. Variations saisonnières de la qualité du sperme chez le bélier lle-de-France. II. Fécondance : relation avec les critères qualitatifs observés in vitro. Reprod. Nutr. Dévelop., 21, 399-407.

COLAS G., 1983. Factors affecting the quality of ram semen, 453-465. In HARESIGN W., Sheep production. Butterworths.

COLAS G., BRICE G., 1976. Seasonal variations of the fertilizing capacity of the deep-frozen ram semen. VIIIth. int. Congr. Anim. Reprod. \& A.l., vol. IV. Krakow, 977-980.

COLAS G., BRICE G., GUERIN Y., 1974. Acquisitions récentes en matière d'Insémination Artificielle ovine. Bull. techn. Inform. Minist. Agric., 294, 795-800.

COLAS G., MÉNISSIER F., COUROT M., PAQUIGNON M., 1984. Technologie de l'insémination artificielle et fertilité du mâle. In Les Colloques de I'I.N.R.A., Edit. I.N.R.A. Versailles (sous presse).

COLAS G., PERSONNIC D., COUROT M., ORTAVANT R.,1975. Influence du rythme de récolte sur la production de spermatozoïdes chez le jeune bélier Romanov. Ann. Zootech., 24, 189198.

COLYER R. J., 1971. Development of the testis and epididymis of the Clun Forest ram. J. agric. Sci. Camb., 76, 433-441. 
COUROT M., TOURNEUR J. C., 1976. Qualité du sperme et réussite de l'insémination artificielle, 117-125. In Maitrise des cycles sexuels chez les bovins, I.N.R.A.-SERSIA Searle, Paris.

DACHEUX J. L., PISSELET Cl., BLANC M. R., HOCHEREAU-de REVIERS, COUROT M., 1981. Seasonal variations in rete testis fluid secretion and sperm production in different breeds of ram. J. Reprod. Fert., 61, 363-371.

FOURNIER-DELPECH S., COLAS G., COUROT M., BRICE G., 1979. Epididymal sperm maturation in the ram : motility, fertilizing ability and embryonic survival after uterine artificial insemination in the ewe. Ann. Biol. anim. Bioch. Biophys., 19, 597-605.

FOWLER D. G., 1965. Semen quality of Merino rams. 2. The effects of seasonal changes in day length on semen quality. Aust. J. exp. Agric. anim. Husb., 5, 247-251.

LANGFORD G. A., MARCUS G. J., 1982. Influence of sperm number and seminal plasma on fertility of progestagen treated sheep in confinement. $J$. Reprod. Fert., 65, 325-329.

LEES J. L., 1971. Some aspects of reproductive efficiency in sheep. Vet. Rec., 88, 86-95.

LINCOLN G. A., 1976. Secretion of LH in rams exposed to two different photoperiods. J. Reprod. Fert., 47, 351-353.

LINCOLN G. A., 1977. Changes in pituitary responsiveness to luteinizing hormone releasing hormone in rams exposed to artificial photoperiods. J. Endocrinol., 73, 519-527.

LINCOLN G. A., DAVIDSON W., 1977. The relationship between sexual and aggressive behaviour, and pituitary and testicular activity during the sexual cycle of rams, and the influence of photoperiod. J. Reprod. Fert., 49, 267-276.

MANN T., 1964. The biochemistry of semen and of the male reproductive tract. Methuen Co. Edit., p. 89.

MICKELSEN D. W., PAISLEY L. G., DAHMEN J. J., 1981. The effect of season on the scrotal circumference and sperm motility and morphology in rams. Theriogenology, 16, 45-50.

NEVES J. P., GÜNZEL A. R., SCHMIDT H., 1980. Einfluss der Jahreszeit auf die Spermaqualität von Merino. Fleischschafen. Zuchthg., 15, 118-125.

OLLIVIER L., LEGAULT C., 1967. L'influence directe du verrat sur la taille et le poids des portées obtenues par insémination artificielle. Ann. Zootech., 16, 247-254.

ORTAVANT R., 1958. Le cycle spermatogénétique chez le bélier. Th. Doct. ès Sci. Paris 127, pp. $\mathrm{N}^{\circ}$ A 3118-03990.

ORTAVANT R., 1977. Photoperiodic regulation of reproduction in the sheep. Proc. Symp. Management of reproduction in sheep and goat. Univ. of Wisconsin, Madison, 58-71.

ORTAVANT R., THIBAULT C., 1956. Influence de la durée quotidienne d'éclairement sur la spermatogenèse du bélier. C. R. Soc. Biol., CL, 358-36.

PELLETIER J., 1971. Influence du photopériodisme et des androgènes sur la synthèse et la libération de $L H$ chez le bélier. Th. Doct. Etat. ès Sciences Nat. Paris CNRS. A.D. 5441.

SALAMON S., ROBINSON T. J., 1962. Studies on the artificial insemination of merino sheep. I. The effects of frequency, of season of insemination, age of the ewe, rams and milk diluents on lambing performance. Aust. J. agri. Res., 13, 52-68.

SANFORD L. M., BEATON B. D., HOWLAND B. E., PALMER M. W., 1978. Photoperiod-induced changes in LH, FSH, prolactin and testosterone secretion in the ram. Can. J. anim. Sci, 58, 123-128.

SCHANBACHER B. D., 1979. Increased lamb production with rams exposed to short day-lengths during the non-breeding season. J. anim. Sci, 49, 927-932.

TULLEY D., BURFENING P. J., 1983. Libido and scrotal circumference of rams as affected by season of the year and altered photoperiod. Theriogenology, 20, 435-448. 